\title{
KERNEL FUNCTORS FOR WHICH THE ASSOCIATED IDEMPOTENT KERNEL FUNCTOR IS STABLE
}

\author{
by J. N. MANOCHA
}

(Received 27 July, 1974; revised 22 March, 1975)

1. Preliminaries. Let $R$ be a ring with unity and let $\mathfrak{M}_{R}$ denote the category of unital right $R$-modules. A preradical $\gamma$ of $\mathfrak{M}_{R}$ is a functor $\gamma: \mathfrak{M}_{R} \rightarrow \mathfrak{M}_{R}$ such that

(i) $\gamma(M) \subseteq M$ for each $R$-module $M$,

(ii) for $f: M \rightarrow N, \gamma(f)$ is the restriction of $f$ to $\gamma(M)$.

$\gamma$ is a radical if (iii) $\gamma(M / \gamma(M))=0$ for all $R$-modules $M$. $\gamma$ is left exact or $\gamma$ is a kernel functor in the sense of Goldman [2] if (iii)' for a submodule $N$ of an $R$-module $M, \gamma(N)=\gamma(M) \cap N$. A left exact radical is nothing but an idempotent kernel functor as defined in [2].

Let $\sigma$ be an idempotent kernel functor. An $R$-module $M$ is said to be $\sigma$-torsion ( $\sigma$-torsionfree) if $\sigma(M)=M(\sigma(M)=0)$. If we denote the classes of $\sigma$-torsion and $\sigma$-torsion-free modules by $\mathscr{T}_{\sigma}$ and $\mathscr{F}_{\sigma}$ respectively, then the pair $\left(\mathscr{T}_{\sigma}, \mathscr{F}_{\sigma}\right)$ is a hereditary torsion theory for $\mathfrak{M}_{R}$. More precisely: a torsion theory for $\mathfrak{M}_{R}$ is a pair $(\mathscr{T}, \mathscr{F})$ of classes of $R$-modules such that

$$
\begin{aligned}
& \mathscr{F}=\left\{N_{R} \mid \operatorname{Hom}_{R}[K, N]=0 \text { for all } K \in \mathscr{T}\right\}, \\
& \mathscr{T}=\left\{M_{R} \mid \operatorname{Hom}_{R}[M, L]=0 \text { for all } L \in \mathscr{F}\right\} .
\end{aligned}
$$

$\mathscr{T}$ is closed under homomorphic images, direct sums and extensions. $\mathscr{F}$ is closed under submodules, direct products and extensions.

The torsion theory $(\mathscr{T}, \mathscr{F}$ ) is said to be hereditary if $\mathscr{T}$ (or equivalently $\mathscr{F}$ ) is closed under submodules (injective envelopes).

We have a one-to-one correspondence between idempotent kernel functors on $\mathfrak{M}_{R}$ and hereditary torsion theories for $\mathfrak{M}_{R}$. The correspondence is given by

$$
\sigma \rightarrow \mathscr{T}_{\sigma}=\left\{M_{R} \mid \sigma(M)=M\right\}
$$

with the inverse correspondence $\mathscr{T} \rightarrow \sigma_{\mathscr{T}}$, where, for an $R$-module $M, \sigma_{\mathscr{T}}(M)=\Sigma\{N \mid N$ is a submodule of $M$ and $N \in \mathscr{T}\}$. For details, we refer the reader to Goldman [2], Lambek [3] and Stenstrom [7].

If $\gamma_{1}$ and $\gamma_{2}$ are preradicals, $\gamma_{1} \leqq \gamma_{2}$ if $\gamma_{1}(M) \subseteq \gamma_{2}(M)$ for all $R$-modules $M$.

For the proof of the following proposition, we refer to Stenstrom [7, Proposition 1.1] or Goldman [2, Proposition 1.1, Theorem 1.6].

PROPOSITION 1.1. With each preradical $\gamma$, one can associate a radical to be denoted by $\bar{\gamma}$, such that

(i) $\gamma \leqq \bar{\gamma}$,

(ii) $\bar{\gamma}$ is a radical,

(iii) if $\mu$ is a radical and $\gamma \leqq \mu$, then $\bar{\gamma} \leqq \mu$.

Moreover, if $\gamma$ is a kernel functor, so is $\bar{\gamma}$. That is, $\bar{\gamma}$ defines an idempotent kernel functor. 
$\bar{\gamma}$ is obtained by transfinite induction as follows: let $M$ be an $R$-module. For a non-limit ordinal $\beta$, define $\gamma_{\beta}$ by $\gamma_{\beta}(M) / \gamma_{\beta-1}(M)=\gamma\left(M / \gamma_{\beta-1}(M)\right)$ and for a limit ordinal $\beta$, define $\gamma_{\beta}$ by $\gamma_{\beta}(M)=\sum_{\alpha<\beta} \gamma_{\alpha}(M)$. This yields an ascending sequence of preradicals. $\bar{\gamma}$ is now given by $\bar{\gamma}(M)=\sum_{\beta}^{\alpha} \gamma_{\beta}(M)$. Equivalently, we can define $\bar{\gamma}(M)=\cap\{N \mid N \subseteq M$ and $\gamma(M / N)=0\}$. We note that $\gamma(M)=0$ implies that $\bar{\gamma}(M)=0$.

2. Main result and applications. Let $\mathscr{E}$ be a class of $R$-modules. By the hereditary torsion class generated by $\mathscr{E}$ is meant the smallest class $\mathscr{T}_{\mathscr{E}}$ containing $\mathscr{E}$ such that $\mathscr{T}_{\mathscr{E}}$ is a hereditary torsion class for some hereditary torsion theory.

LEMMA 2.1. Let $\gamma$ be a kernel functor, $\mathscr{E}_{\gamma}=\{M \mid \gamma(M)=M\}$ and $\mathscr{T}_{\mathscr{B}_{\gamma}}$ the hereditary torsion class generated by $\mathscr{E}_{\gamma}$. Then $\mathscr{T}_{\mathscr{E}_{\gamma}}=\mathscr{T}_{\bar{\gamma}}$, where $\mathscr{T}_{\bar{\gamma}}$ is the class of torsion modules corresponding to the idempotent kernel functor $\bar{\gamma}$.

Proof. $\bar{\gamma}$ is the smallest idempotent kernel functor larger than $\gamma$, by Proposition 1.1. Since there is a one-to-one correspondence between idempotent kernel functors on $\mathfrak{M l}_{R}$ and hereditary torsion theories for $\mathfrak{M}_{R}, \mathscr{T}_{\bar{\gamma}}$ must correspond to the smallest hereditary torsion class containing $\mathscr{E}_{\gamma}$. Thus $\mathscr{T}_{\varepsilon_{\gamma}}=\mathscr{T}_{\bar{\gamma}}$.

LEMMA 2.2. Let $\gamma$ be a kernel functor. Then for each $R$-module $M, \gamma(M)$ is an essential submodule of $\bar{\gamma}(M)$.

Proof. Let $N \subseteq \bar{\gamma}(M)$ be such that $N \cap \gamma(M)=0$. We show that $N=0$. Since $\gamma$ is a kernel functor, $\gamma(N)=N \cap \gamma(M)=0$. This implies that $\bar{\gamma}(N)=0$. But $N \subseteq \bar{\gamma}(M)$ and hence $\bar{\gamma}(N)=N$. Thus $N=0$ and the lemma follows.

DefinItion 2.3. A hereditary torsion theory $(\mathscr{T}, \mathscr{F})$ is said to be stable if $\mathscr{T}$ is closed under essential extensions. We shall call an idempotent kernel functor $\sigma$ stable if the corresponding hereditary torsion theory is stable. (See Stenstrom [7, §4] and Gabriel [1].)

THEOREM 2.4. Let $\gamma$ be a kernel functor such that $\bar{\gamma}$ is stable. Then the following statements are equivalent.

(i) $\gamma(R)$ is an essential right ideal of $R$.

(ii) $\gamma(M)$ is an essential submodule of $M$ for all $R$-modules $M$.

(iii) If $M \neq 0$, then $\gamma(M) \neq 0$.

(iv) $\bar{\gamma}(R)=R$.

(v) $\bar{\gamma}(M)=M$ for all $M$; that is $\bar{\gamma}$ is the identity functor on $\mathfrak{M}_{R}$.

(vi) Each hereditary torsion theory for $\mathfrak{M}_{R}$ is generated by a class of $\gamma$-torsion modules. (Here an $R$-module $M$ is $\gamma$-torsion if $\gamma(M)=M$.) 
Proof. $\quad(i) \Rightarrow(i v) . \quad \bar{\gamma}(\gamma(R))=\gamma(R)$. Since $\bar{\gamma}$ is stable, we have $\bar{\gamma}(R)=R$.

(iv) $\Rightarrow(v)$. Since $\mathscr{T}_{\bar{\gamma}}$ is closed under homomorphic images and direct sums, $\bar{\gamma}(R)=R$ implies that $\bar{\gamma}(M)=M$ for all $M$.

$(v) \Rightarrow(i v)$. Trivial.

$(v) \Rightarrow(i i)$. By Lemma 2.2, $\gamma(M)$ is an essential submodule of $\bar{\gamma}(M)$. Thus since $\bar{\gamma}(M)=M$, the implication follows.

(ii) $\Rightarrow($ iii $)$. Trivial.

(iii) $\Rightarrow(i)$. Suppose not. Then there exists a non-zero right ideal $I$ such that $\gamma(R) \cap I=0$. Now $\gamma(I) \subseteq \gamma(R)$. This implies that $\gamma(I)=0$, a contradiction.

$(v) \Rightarrow(v i)$. The class $\mathscr{E}=\{M \mid \gamma(M)=M\}$ is closed under submodules and factor modules. By Lemma 2.1, the hereditary torsion class generated by $\mathscr{E}$ is all of $\mathfrak{M i}_{R}$. Now let $\mathscr{T}$ be a hereditary torsion class. Then by Stenstrom [7, Exercise 3, p. 11] $\mathscr{T}$ is generated by $\mathscr{T} \cap \mathscr{E}$. That is $\mathscr{T}$ is generated by a class of $\gamma$-torsion modules.

$(v i) \Rightarrow(v)$. Take $\mathscr{T}=\mathfrak{M}_{R}$. Then $\mathfrak{M}_{R}$ is generated by a class of $\gamma$-torsion modules. By Lemma $2.1, \bar{\gamma}$ is the identity functor on $\mathfrak{M}_{R}$. Thus $\bar{\gamma}(M)=M$ for all $R$-modules $M$.

Let $R$ be a ring and $M$ an $R$-module. The singular submodule of $M$, to be denoted by $Z_{R}(M)$, is the set of all elements of $M$ which are annihilated by essential right ideals of $R$. $Z_{R}\left(\right.$ ) defines a kernel functor on $\mathfrak{M}_{R}$. The idempotent kernel functor corresponding to $Z_{R}()$ is called the Goldie torsion functor. It will be denoted by $\mathscr{G}$.

We note that the Goldie torsion class is generated by the class of modules of the form $A / B$, where $A$ is an essential extension of $B$. Moreover, the Goldie torsion functor is stable.

As a special case of Theorem 2.4 we have the following result.

Proposition 2.5. Let $R$ be a ring. Then the following statements are equivalent.

(i) $Z_{R}\left(R_{R}\right)$ is an essential right ideal of $R$.

(ii) $Z_{R}(M)$ is an essential submodule for each $R$-module $M$.

(iii) $Z_{R}(M) \neq 0$ for every non-zero $R$-module $M$.

(iv) $\mathscr{G}(R)=R$.

(v) $\mathscr{G}(M)=M$ for each $R$-module $M$.

(vi) Each hereditary torsion theory for $\mathfrak{M}_{R}$ is generated by a class of singular modules.

REMARK. Using different methods, Ming [4] has also established the equivalence of (i), (ii) and (iii).

Proposition 2.6. Let $R$ be a commutative noetherian ring and let $\gamma$ be a kernel functor. Then the following statements are equivalent.

(i) $\gamma(R)$ is an essential ideal of $R$.

(ii) For each $R$-module $M, \gamma(M)$ is an essential submodule of $M$.

(iii) $\gamma(M) \neq 0$ for a non-zero module $M$.

(iv) Each hereditary torsion theory for $\mathfrak{M}_{R}$ is generated by a class of $\gamma$-torsion modules.

Proof. By a result of Gabriel [1], every hereditary torsion class for a commutative noetherian ring is stable. The result follows from Theorem 2.4. 
As an application we have

Proposition 2.7. Let $R$ be a commutative noetherian ring. Then the following statements are equivalent.

(i) Socle $(R)$ is an essential ideal of $R$.

(ii) $R$ is an artinian ring.

(Here, for an $R$-module $M$, Socle $(M)$ is the sum of all simple submodules of $M$.)

Proof. (i) $\Rightarrow$ (ii). Socle () defines a kernel functor on $\mathfrak{D}_{R}$. From the last theorem, Socle $(M) \neq 0$ for each non-zero module $M$. Define an ascending sequence of ideals as follows: $I_{0}=\operatorname{Socle}(R)$ and $I_{n+1} \supseteq I_{n}$ with $I_{n+1} / I_{n}=$ Socle $\left(R / I_{n}\right)$. Either $R=I_{m}$ for some $m$ or we get a strictly ascending sequence $I_{0} \subseteq I_{1} \subseteq \ldots$, since Socle $\left(R / I_{n}\right) \neq 0$. Since $R$ is noetherian, this sequence terminates, say at $m$. Thus $R=I_{m}$ for some integer $m$. Now $I_{n+1} / I_{n}$ has finite length for each $n$. Hence $R$ itself is of finite length. Thus $R$ is artinian.

$($ ii) $\Rightarrow(i)$. Trivial.

REMARK. The above sharpens a result of Nita in [6] where, using different methods, the above equivalence is proved assuming further that $R$ is an $S$-ring in the sense of Morita [5].

\section{REFERENCES}

1. P. Gabriel, Des catégories abeliennes, Bull. Soc. Math. France 90 (1962), 323-448.

2. O. Goldman, Rings and modules of quotients, J. Algebra 13 (1969), 10-47.

3. J. Lambek, Torsion theories, additive semantics and rings of quotients, Lecture Notes in Mathematics No. 177 (Springer-Verlag, New York/Berlin, 1971).

4. R. Yue Che Ming, A note on singular ideals, Tohoku Math. J. 21 (1969), 337-342.

5. K. Morita, On S-rings, Nagoya Math. J. 27 (1966), 687-695.

6. M. C. Nita, Sur les anneaux A tells que tout A-module simple est isomorphic à un ideal, C. R. Acad. Paris 268 (1969), 88-91.

7. B. Stenstrom, Rings and modules of quotients, Lecture Notes in Mathematics No. 237 (SpringerVerlag, New York/Berlin, 1971).

Kent State University

EAST Liverpool CAMPUS

EAST LIVERPOOL, ОhIO 43920, U.S.A. 